\title{
MOVING OBJECT DETECTION USING SPATIAL CORRELATION IN LAB COLOUR SPACE
}

\author{
A. Roshan ${ }^{1, *}$, Y. Zhang ${ }^{1}$ \\ ${ }^{1}$ Department of Geodesy and Geomatics Engineering, University of New Brunswick Fredericton, New Brunswick, Canada \\ (aroshan@unb.ca, yunzhang@unb.ca)
}

Commission II, WG II/5

KEY WORDS: Moving Object Detection, Background Subtraction, Lab Colour Space, Spatial Correlation

\begin{abstract}
:
Background subtraction-based techniques of moving object detection are very common in computer vision programs. Each technique of background subtraction employs image thresholding algorithms. Different thresholding methods generate varying threshold values that provide dissimilar moving object detection results. A majority of background subtraction techniques use grey images which reduce the computational cost but statistics-based image thresholding methods do not consider the spatial distribution of pixels. In this study, authors have developed a background subtraction technique using Lab colour space and used spatial correlations for image thresholding. Four thresholding methods using spatial correlation are developed by computing the difference between opposite colour pairs of background and foreground frames. Out of 9 indoor and outdoor scenes, the object is detected successfully in 7 scenes whereas existing background subtraction technique using grey images with commonly used thresholding methods detected moving objects in 1-5 scenes. Shape and boundaries of detected objects are also better defined using the developed technique.
\end{abstract}

\section{INTRODUCTION}

A moving object is extracted from video frames using moving object detection techniques. The detected object is used in traffic monitoring, security surveillance, site monitoring, face detection, military applications, photography, and robotics (Kamate \& Yilmazer, 2015; Kumar, et al., 2016). Moving object detection techniques can be divided into two major categories: Background-based and Frame-based techniques. Backgroundbased techniques use static background and detect the moving object by processing it with the incoming frame (Piccardi, 2004). Two or more consecutive frames are used in frame-based techniques. Among all the background-based techniques, background subtraction using grey images is the most commonly used technique. A detailed literature on moving object detection techniques is available (McIvor, 2000; Shaikh, et al., 2014; Piccardi, 2004; Hu, et al., 2004). Further, due to noise present in the camera scene, different background modeling methods are introduced to model the background but background modeling comes with a computing cost. Some techniques also use $R G B$ scheme for scene processing (Chun-yang, et al., 2013; Cucchiara, et al., 2003; Nummiaro, et al., 2003; Horprasert , et al., 1999; Carmona , et al., 2008). Researchers have also utilized $H S V, L U V$, and $L a b$ colour spaces to detect moving objects and eliminate shadows detected with the moving objects (Shan, et al., 2007; Chen \& Lei, 2004; El Baf, et al., 2008). Application of $R G B$ bands is also less relevant because the pixel values in $R G B$ bands are correlated (Piva, et al., 1999).

In background subtraction techniques, an incoming frame (or foreground frame) is subtracted from the background frame and the difference is subjected to a threshold value. Unwanted noise is removed using a threshold. Image thresholding is an integral part of moving object detection as it is used with every technique to extract moving object out of noisy frames (McIvor, 2000; Sezgin \& Sankur, 2004). There exist different thresholding methods such as Otsu threshold (Otsu, 1979), adaptive threshold (Bradley \& Roth, 2007; Wellner, 1993), iterative Gaussian clustering (Ridler \& Calvard, 1978; Şahan, et al., 2016), histogram based threshold (Glasbey, 1993; Kapur, et al., 1985; Carabias, 2012), and minimum error threshold (Kittler \& Illingworth, 1986), but it is difficult to find a method which works under different scene conditions. Different methods provide different threshold values which result into varying outputs for moving object detection. A wrong threshold value results in partial or complete disappearance of the object.

This research paper is focused on the improvement of a background subtraction technique by incorporating colour bands of opposite colour pairs and introducing image thresholding using spatial segmentation. A new background subtraction technique using $L a b$ colour space is proposed in which correlated $R G B$ colour space bands are converted to uncorrelated $L a b$ colour space (L: lightness, a: red-green opposite colour pair, and b: blue-yellow opposite colour pair). Opposite colour pairs in the background are subtracted from those in the foreground. Effect of change in scene light is reduced by using opposite colour pairs in this technique. Furthermore, four different automatic image thresholding techniques using spatial correlation are also developed as statistics based thresholding methods do not consider the spatial distribution of pixels. Output from grey image based moving object detection technique using existing automatic image thresholding is compared with results from $L a b$ colour space background subtraction using spatial correlation based automatic image thresholding.

\section{METHODOLOGY}

Video frames in $R G B$ colour space are transformed into $L a b$ colour space (Alessi, et al., 2004). Opposite colour pairs of the background are subtracted from opposite colour pairs of incoming frames. Spatial correlation between the differences is calculated which is subjected to threshold as described in Figure 1. Effect of light change in the camera scene is reduced by ignoring lightness component of the Lab colour space 


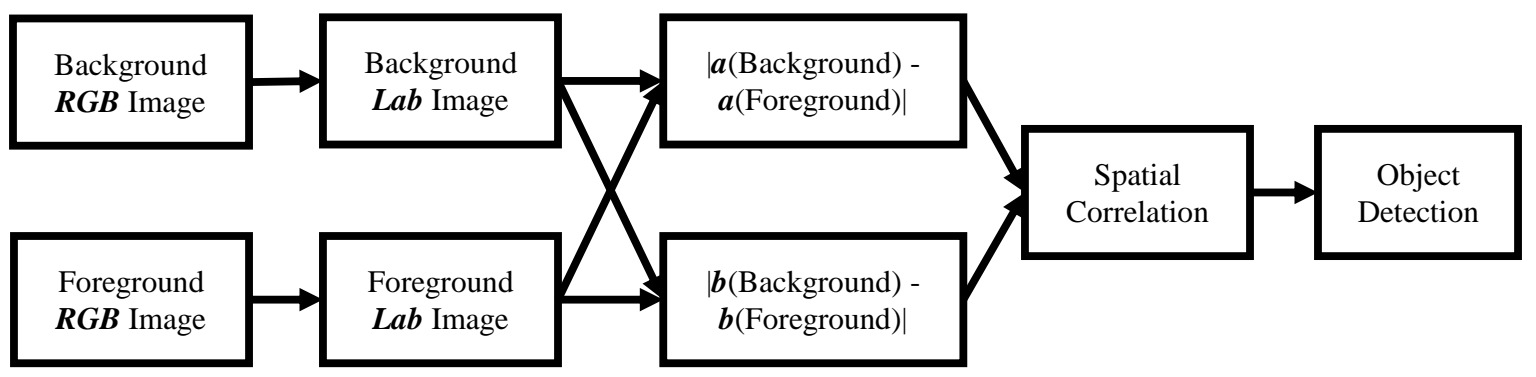

Figure 1. Moving object detection using Lab colour space and spatial correlation

\subsection{Background and Foreground Subtraction}

$L a b$ colour bands of background and foreground images are subtracted and the difference bands of $\mathrm{L}, \mathrm{a}$, and $\mathrm{b}$ are computed (eqn. 1). The difference between background and foreground frames is further processed for moving object detection.

$$
\begin{aligned}
\Delta L & =\left|L_{\text {Background }}-L_{\text {Foreground }}\right| \\
\Delta a & =\left|a_{\text {Background }}-a_{\text {Foreground }}\right| \\
\Delta b & =\left|b_{\text {Background }}-b_{\text {Foreground }}\right|
\end{aligned}
$$

\subsection{Spatial Correlation}

With the assumption that the moving object is present in both the differences of opposite colour pairs (eqn. 1), physical location of pixels corresponding to moving object should overlap in both the differences. Spatial correlation between $\Delta \mathrm{a}$ and $\Delta \mathrm{b}$ is determined. Three different methods of spatial correlation are used to detect the moving object. These methods are Moran's Index (Anselin, 1995), Jaccard's Index (Jaccard, 1912), and cross-correlation (Proakis \& Manolakis, 1996; MathWorks, 2018). The coefficients at each pixel location is calculated using window operations as shown in Figure 2.

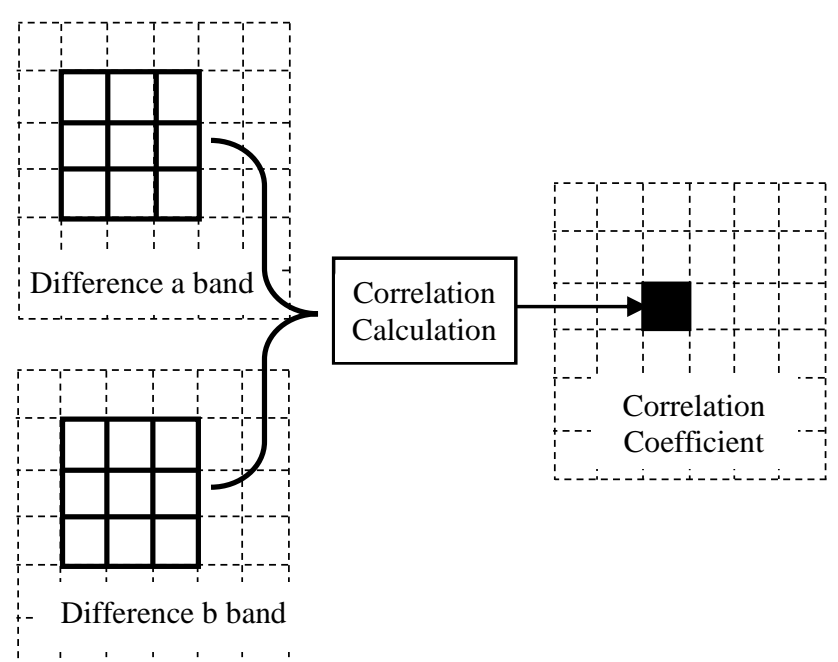

Figure 2. Spatial correlation calculation between two images

\subsubsection{Windowed Operation to Calculate Coefficient} Indices: Differences of opposite colour pairs are divided into small segments using a moving window of size $3 \times 3$ (or a higher odd number). Thereafter, correlation index between windowed segments is calculated (Figure 2). This way a matrix consisting of correlation indices is created. This process is repeated for different widow sizes and finally all the matrices are summed to obtain the final matrix consisting of correlation indices.
2.2.2 Sliding and Non-Sliding Window Operations: In a sliding window operation, the window moves from one pixel location to the next and the calculated coefficient index is assigned to the pixel corresponding to the central position of the moving window. In a non-sliding window operation, the window jumps from one pixel location to another such that it does not overlap from one location to another and the correlation index is assigned to all pixel locations within the window. Moran's and Jaccard indices are calculated using sliding window operation whereas cross-correlation coefficients are calculated using both the sliding and non-sliding window operations.

\subsection{Coefficient Thresholding}

A moving object is detected by thresholding coefficient matrices. An image threshold is applied to all positive coefficients and a pixel is marked as a moving object only if the correlation coefficient is greater than the threshold coefficient value (eqn. 2) which is obtained using the conditions given in Table 1. Different threshold criterion are selected for different methods of coefficient calculation because they use different statistics.

Moving Object Pixel $=$

$\{$ Yes, if Correlation Coefficient $>$ Th

No, Otherwise

\begin{tabular}{|l|l|}
\hline \multicolumn{1}{|c|}{ Coefficient } & \multicolumn{1}{c|}{ Threshold } \\
\hline $\begin{array}{l}\text { Cross-Correlation } \\
\text { (sliding window) }\end{array}$ & $\begin{array}{l}0.1 \\
\times \max \text { (Correlation Coefficient })\end{array}$ \\
\hline $\begin{array}{l}\text { Cross-Correlation } \\
\text { (distinct window) }\end{array}$ & $\begin{array}{l}0.1 \\
\times \max (\text { Correlation Coefficient })\end{array}$ \\
\hline Moran's Index & $0.1 \times \max ($ Moran's Index $)$ \\
\hline Jaccard Index & $0.9 \times \max$ (Jaccard Index $)$ \\
\hline
\end{tabular}

Table 1. Threshold selection criteria using spatial correlation coefficients

\section{RESULTS AND DISCUSSIONS}

\subsection{Dataset}
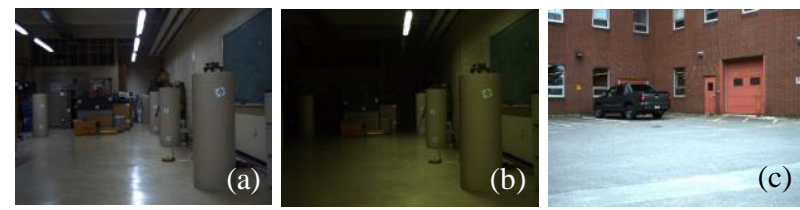

Figure 3. Indoor and outdoor camera scenes

The video dataset with a single moving object is collected in both indoor and outdoor conditions. For indoor conditions, an object starts moving from far end towards the camera in a long hallway with regular fluorescent lights. The lights were adjusted to collect data in two different light conditions as High and Medium 
light (Figure 3a, b). A parking lot is selected as an outdoor scene where natural sun light is the light source. The moving object and camera scenes were not exposed to direct sunlight (Figure 3c)

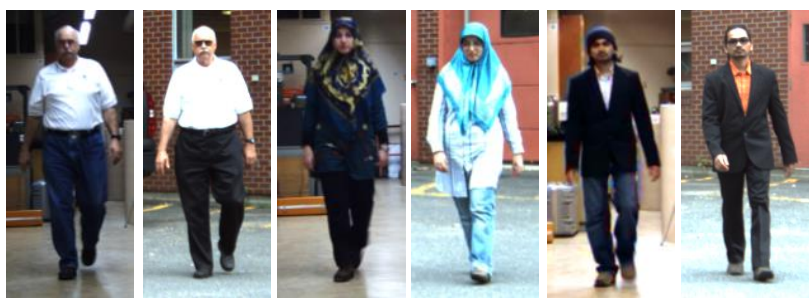

Figure 4. Moving objects in indoor and outdoor scenes (from left to right: Object $1\left(\mathrm{O}_{1}\right)$ indoor, Object 1 outdoor, Object 2 $\left(\mathrm{O}_{2}\right)$ indoor, Object 2 outdoor, Object $3\left(\mathrm{O}_{3}\right)$ indoor and Object 3 outdoor
In order to test the developed moving object detection technique, videos with distinct moving objects are captured. Objects are differentiated in terms of their shape and appearance (Figure 4).

\subsection{Moving Object Detection}

Figure 5, Figure 7, and Figure 6 show outputs from the developed methodology (section 2) of moving object detection. Results from the developed methodology are compared with other commonly used background subtraction techniques using different image thresholding methods. Each column in these figures shows results from different methods. In Figures 5-7, columns from left to right depict, 1) cross correlation using sliding window, 2) cross correlation using distinct window, 3) Moran's index,4) Jaccard coefficient, 5) Otsu, and 6) Adaptive thresholding. Six different existing thresholding methods (iterative Gaussian, entropy, minimum error thresholding,

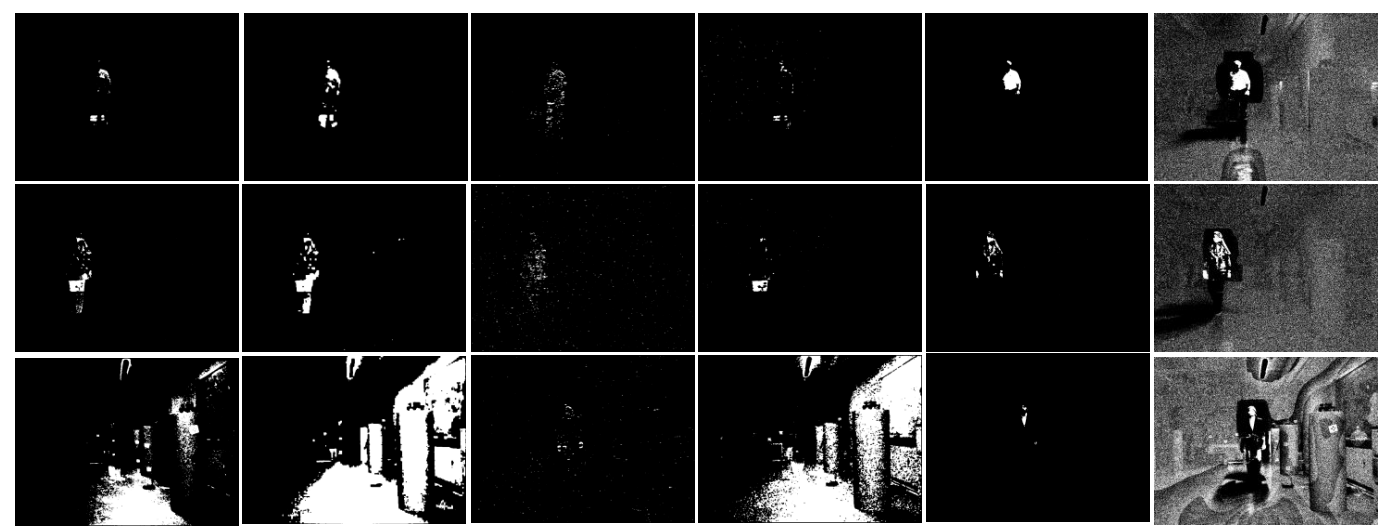

Figure 5. Moving object detection in indoor medium light scene (Row 1: Object 1, Row 2: Object 2, Row 3: Object 3)

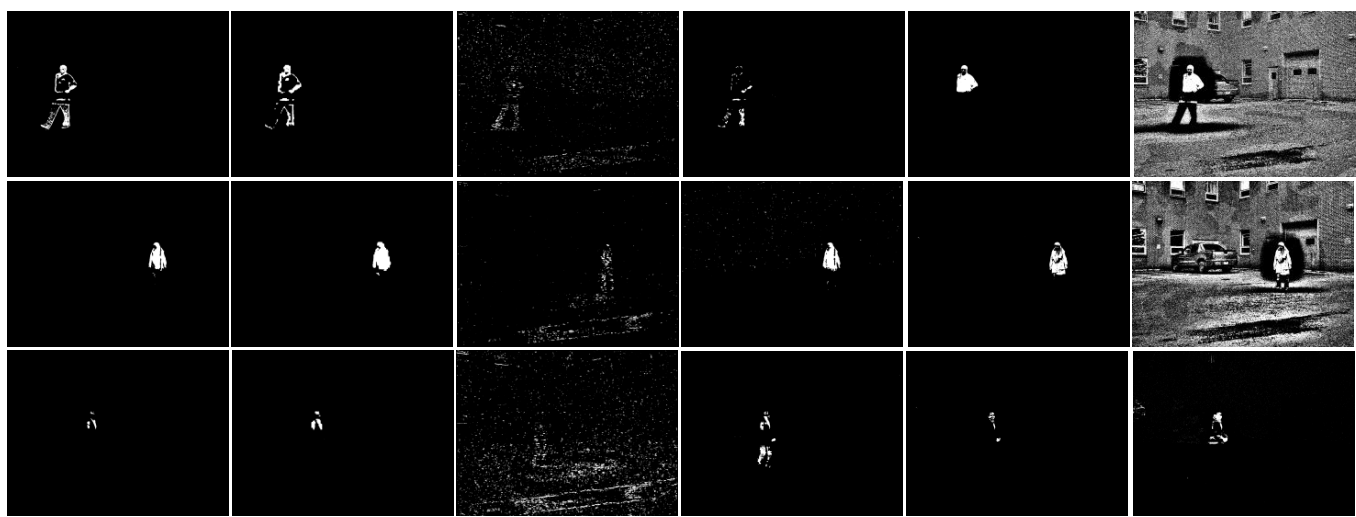

Figure 6. Moving object detection in day light outdoor scene (Row 1: Object 1, Row 2: Object 2, Row 3: Object 3)

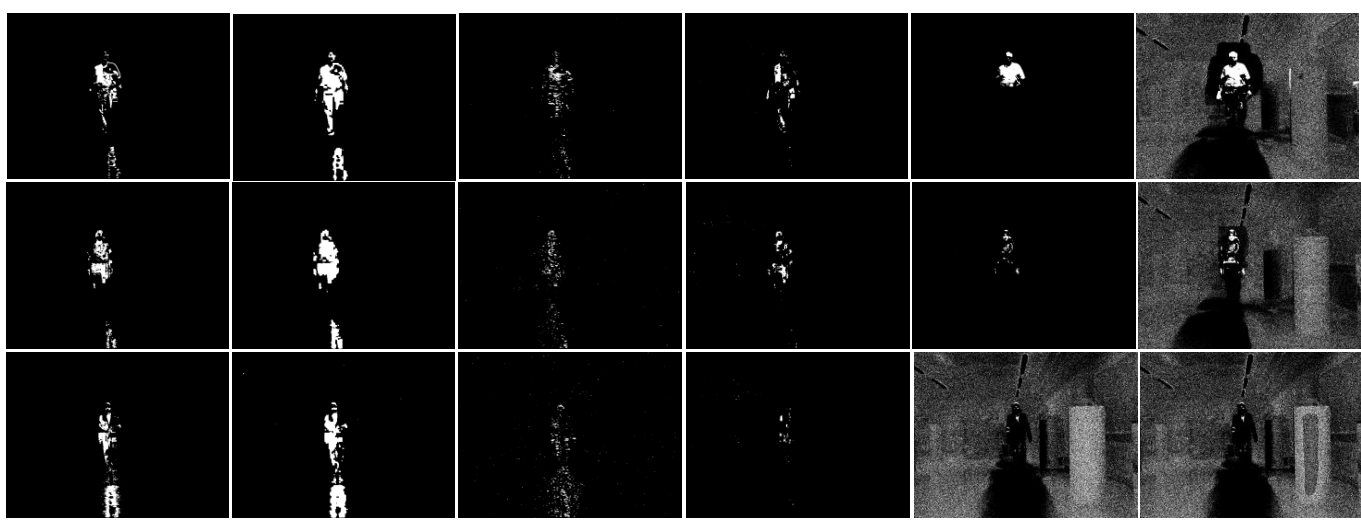

Figure 7. Moving object detection in indoor high light scene (Row 1: Object 1, Row 2: Object 2, Row 3: Object 3) 
moving average, Otsu, and Adaptive thresholding) are used with the background subtraction technique using grey images. Among the six methods, only Otsu and Adaptive thresholding detected the moving object.

It can be observed that moving object detection failed using the Adaptive thresholding technique (last column in Figure 5-Figure 6 ). An object of a darker shade of colour could not be detected (Figure 5, row 3) in indoor medium light conditions. However, other objects of lighter shades are detected under similar light conditions (Figure 5, row 1 and 2). The effect of light conditions in a camera scene is demonstrated in Figure 7 (row 3 ) where an object of darker shade was detected in indoor high light conditions. Object detection rate using Otsu and Adaptive thresholding techniques is lower than techniques using $L a b$ colour space and spatial segmentation. Both Otsu and Adaptive thresholding-based techniques failed to detect the moving object as Otsu thresholding detects

\begin{tabular}{|l|l|l|l|l|l|l|l|l|l|l|l|}
\hline \multirow{2}{*}{ Thresholding Method } & \multicolumn{3}{|c|}{$\begin{array}{c}\text { Indoor } \\
\text { Medium Light }\end{array}$} & \multicolumn{4}{|c|}{$\begin{array}{c}\text { Indoor High } \\
\text { Light }\end{array}$} & \multicolumn{3}{c|}{$\begin{array}{c}\text { Outdoor } \\
\text { Daylight }\end{array}$} & \multirow{2}{*}{$\begin{array}{c}\text { Yes } \\
\text { /No }\end{array}$} \\
\cline { 2 - 12 } & $\mathbf{O}_{\mathbf{1}}$ & $\mathbf{O}_{\mathbf{2}}$ & $\mathbf{O}_{\mathbf{3}}$ & $\mathbf{O}_{\mathbf{1}}$ & $\mathbf{O}_{\mathbf{2}}$ & $\mathbf{O}_{\mathbf{3}}$ & $\mathbf{O}_{\mathbf{1}}$ & $\mathbf{O}_{\mathbf{2}}$ & $\mathbf{O}_{\mathbf{3}}$ & \\
\hline $\begin{array}{l}\text { 1. Spatial Correlation } \\
\text { using sliding window }\end{array}$ & $\mathrm{Y}$ & $\mathrm{Y}$ & $\mathrm{N}$ & $\mathrm{Y}$ & $\mathrm{Y}$ & $\mathrm{Y}$ & $\mathrm{Y}$ & $\mathrm{Y}$ & $\mathrm{N}$ & $7 / 2$ \\
\hline $\begin{array}{l}\text { 2. Spatial Correlation } \\
\text { using distinct window }\end{array}$ & $\mathrm{Y}$ & $\mathrm{Y}$ & $\mathrm{N}$ & $\mathrm{Y}$ & $\mathrm{Y}$ & $\mathrm{Y}$ & $\mathrm{Y}$ & $\mathrm{Y}$ & $\mathrm{N}$ & $7 / 2$ \\
\hline 3. Moran's Index & $\mathrm{Y}$ & $\mathrm{Y}$ & $\mathrm{N}$ & $\mathrm{Y}$ & $\mathrm{Y}$ & $\mathrm{N}$ & $\mathrm{N}$ & $\mathrm{N}$ & $\mathrm{N}$ & $4 / 5$ \\
\hline 4. Jaccard Index & $\mathrm{N}$ & $\mathrm{N}$ & $\mathrm{N}$ & $\mathrm{Y}$ & $\mathrm{Y}$ & $\mathrm{N}$ & $\mathrm{Y}$ & $\mathrm{Y}$ & $\mathrm{Y}$ & $5 / 4$ \\
\hline 5. Otsu Threshold & $\mathrm{N}$ & $\mathrm{N}$ & $\mathrm{N}$ & $\mathrm{Y}$ & $\mathrm{Y}$ & $\mathrm{N}$ & $\mathrm{Y}$ & $\mathrm{Y}$ & $\mathrm{Y}$ & $5 / 4$ \\
\hline 6. Adaptive Threshold & $\mathrm{N}$ & $\mathrm{N}$ & $\mathrm{N}$ & $\mathrm{N}$ & $\mathrm{N}$ & $\mathrm{N}$ & $\mathrm{N}$ & $\mathrm{N}$ & $\mathrm{Y}$ & $1 / 8$ \\
\hline
\end{tabular}
partial object and Adaptive thresholding detects background with partial object. Although Otsu thresholding failed to completely detect the object, whenever the contrast between background and foreground was better, Otsu thresholding resulted in better object detection (only upper body of the moving object) than when the contrast was poor (Figure 5-Figure $6)$.

In the indoor and outdoor, medium and high light conditions, spatial correlation-based image thresholding gave better moving object detection results than Otsu or Adaptive thresholdingbased detection. Figure 8 shows a larger picture of a detected object (left-most object in Figure 4) in all scenes and light conditions. Each column of Figure 8 shows moving object detection results from (left to right) cross correlation using sliding window, cross correlation using distinct window, Moran's index, Jaccard coefficient, and Otsu thresholding. Spatial correlation-based techniques detected a complete moving object in comparison to a partial detection using Otsu thresholding.
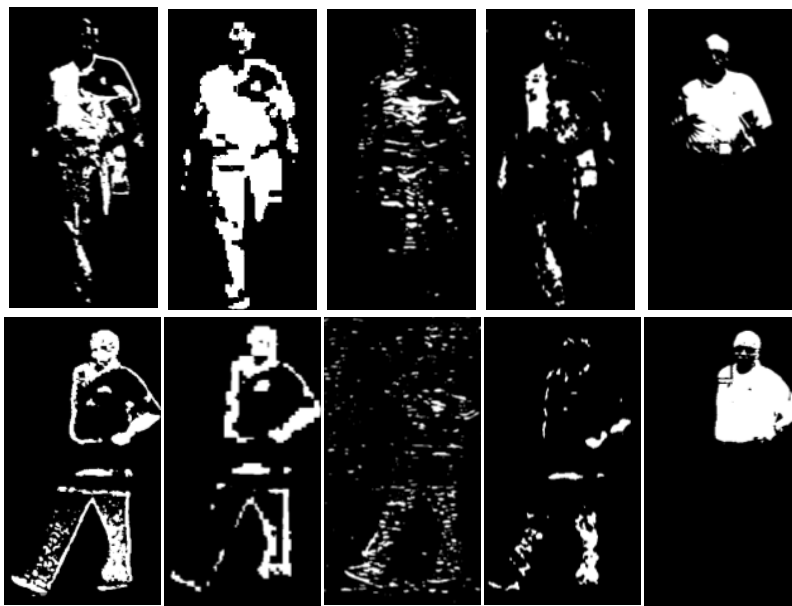

Figure 8. Detailed detected object in indoor and outdoor scenes

A comparison of moving object detection results from Figure 5 Figure 6 is given in Table 2. The last column in the table gives the total number of camera scenes where a moving object was detected vs not detected. Among the four spatial correlationbased techniques, thresholding with cross-correlation using sliding and distinct windows gives better results than thresholding using Moran's and Jaccard indexes. Boundary and shape of the object are also better defined when the object is detected using cross-correlation thresholding. Moran's and Jaccard index-based thresholding results are noisier than crosscorrelation. Further investigation of results leads to the conclusion that shapes and boundaries of detected objects are better defined when the threshold is calculated using crosscorrelation distinct window operation (column 2, Figure 8)

Table 2. Comparison of moving object detection results using six different techniques of image thresholding

\section{CONCLUSIONS}

Moving object detection results are affected by the image threshold value used in extracting the moving object from a camera scene. Different image thresholding techniques give different threshold values which result in the detection of moving objects at various levels. Most image thresholding techniques use grey images converted from $R G B$ images. As $R, G$, and $B$ bands are correlated, moving object detection is not feasible. A new background subtraction technique using un-correlated $L a b$ colour space is developed and the image threshold is determined using spatial correlation. A matrix consisting of spatial correlation indices is calculated and the moving object is detected by using the thresholding correlation coefficients with the highest correlation. Results from the developed techniques are better than the results obtained from Otsu and Adaptive thresholding-based background subtraction techniques. Four spatial correlation determination methods are developed where two of them are based on auto-correlation and the other two are Moran's and Jaccard indices. The most commonly used Otsu image thresholding method failed to detect the complete object under different light and scene conditions. Spatial crosscorrelation determination using distinct window gave better results in terms of complete detection of the object and its boundary when compared with results from cross-correlation using sliding window, Moran's Index, and Jaccard Index. In low light and under poor contrast conditions between foreground and background, all the methods under consideration failed to detect the moving object. Techniques developed in this study also detected shadows of the moving object. In future, frames with poor foreground-background contrast and shadows can be processed further to detect the moving object and remove shadows by implementing shadow removal techniques.

\section{ACKNOWLEDGEMENT}

This research was sponsored by the Atlantic Innovation Fund (AIF) of the Atlantic Canada Opportunities Agency (ACOA) with Yun Zhang as the Principal Investigator. 


\section{REFERENCES}

Alessi, P. J. et al., 2004. CIE 15: Technical Report: Colorimetry. 3rd edition, Washington, D. C.: International Commission on Illumination.

Anselin, L., 1995. Local Indicators of Spatial Association-LISA. Geographical Analysis, 4, 27(2), pp. 93-115.

Bradley, D. \& Roth, G., 2007. Adaptive Thresholding using the Integral Image. Journal of Graphics Tools, 12(2), pp. 13-21.

Carabias, D. M., 2012. Analysis of Image Thresholding Methods for Their Application to Augmented Reality Environments, Madrid: s.n.

Carmona , E. J., Martínez-Cantos , J. \& Mira , J., 2008. A new video segmentation method of moving objects based on bloblevel knowledge. Pattern Recognition Letters, 29(3), pp. 272285.

Chen, B. \& Lei, Y., 2004. Indoor and outdoor people detection and shadow suppression by exploiting HSV color information. s.l., IEEE, pp. 137-142.

Chun-yang, L., Dao-zheng, H. \& Chang-an, L., 2013. Study on Moving Object Detection Based on RGB Color Model. Advanced Materials Research, Volume 710, pp. 700-703.

Cucchiara, R., Grana, C., Piccardi, M. \& Prati, A., 2003. Detecting moving objects, ghosts, and shadows in video streams. IEEE transactions on pattern analysis and machine intelligence.

El Baf, F., Bouwmans, T. \& Vachon, B., 2008. Fuzzy integral for moving object detection. s.1., IEEE, pp. 1719-1736.

Glasbey, C. A., 1993. An Analysis of Histogram-Based Thresholding Algorithms. CVGIP: Graphical Models and Image Processing, 55(6), pp. 532-537.

Horprasert, T., Harwood , D. \& Davis , L. S., 1999. A statistical approach for real-time robust background subtraction and shadow detection. IEEE iccv, 99(1999), pp. 1-19.

Hu, W., Tan, T., Wang, L. \& Maybank, S., 2004. A survey on visual surveillance of object motion and behaviors. IEEE Transactions on Systems, Man, and Cybernetics, Part C (Applications and Reviews), 34(3), pp. 334-352.

Jaccard, P., 1912. The distribution of the flora in the alpine zone. The New Phytologist, 2, 11(2), pp. 37-50.

Kamate, S. \& Yilmazer, N., 2015. Application of Object Detection and Tracking Techniques for Unmanned Aerial Vehicles. Procedia Computer Science, Volume 61, pp. 436-441.

Kapur, J. N., Sahoo, P. K. \& Wong, A. K. C., 1985. A new method for gray-level picture thresholding using the entropy of the histogram. Computer Vision, Graphics, and Image Processing, 29(3), pp. 273-285.

Kittler, J. \& Illingworth, J., 1986. Minimum error thresholding. Pattern Recognition, 19(1), pp. 41-47.

Kumar, P., Singhal, A., Mehta, S. \& Mittal, A., 2016. Real-time moving object detection algorithm on high-resolution videos using GPUs. Real-Time Image Processing, 11(1), pp. 93-109.
MathWorks, 2018. PDF Documentation for Signal Processing Toolbox.

[Online]

Available at: https://www.mathworks.com/help/pdf_doc/signal/signal_ref.pd f [Accessed 308 2018].

McIvor, A., 2000. Background Subtraction Technique. New Zealand, s.n., pp. 147-153.

Nummiaro, K., Koller-Meier, E. \& Gool, L. V., 2003. An adaptive color-based particle filter. Image and Vision Computing, 21(1), pp. 99-110.

Otsu, N., 1979. A Threshold Selection Method from Gray-Level Histograms. IEEE Transactions on Systems, Man, and Cybernetics, 9(1), pp. 62-66.

Piccardi, M., 2004. Background subtraction techniques: a review. 2004 IEEE International Conference on Systems, Man and Cybernetics (IEEE Cat. No.04CH37583), Volume 4, pp. 3099-3104.

Piva, A., Bartolinin, F., Cappellini, V. \& Barni, M., 1999. Exploiting the cross-correlation of RGB-channels for robust watermarking of color images. Kobe, s.n., pp. 306-310.

Proakis, J. G. \& Manolakis, D. G., 1996. Digital Signal Processing: Principles, Algorithms, and Applications. 3 ed. New Jersey: Prentice-Hall International Inc.

Ridler, T. W. \& Calvard, S., 1978. Picture Thresholding Using an Iterative Selection Method. IEEE Transactions on Systems, Man, and Cybernetics, 8, 8(8), pp. 630-632.

Şahan, Ş., Değirmenci, A. \& Çankaya, I., 2016. A Study on Clustering based Image Thresholding Techniques with MATLAB GUI. International Journal of Engineering Science and Computing, 8.pp. 2152-2160.

Sezgin, M. \& Sankur, B., 2004. Survey over image thresholding techniques and quantitative performance evaluation. Journal of Electronic Imaging, 1, 13(1), pp. 146-168.

Shaikh, S. H., Saeed, K. \& Chakri, N., 2014. Moving object detection using background subtraction. In: Moving object detection, approaches, challenges and object tracking. s.1.:Springer, pp. 5-14.

Shan, Y., Yang, F. \& Wang, R., 2007. Color Space Selection for Moving Shadow Elimination. s.l., IEEE, pp. 496-501.

Wellner, P. D., 1993. Adaptive Thresholding for the DigitalDesk, Cambridge: Rank Xerox Ltd 1993. 